\title{
Evaluation of the Volumetric Erosion of Spherical Electrical Contacts Using the Defect Removal Method
}

\author{
Defen Zhang, Martyn Hill, and John W. McBride
}

\begin{abstract}
Volumetric erosion is regarded as a significant index for studying the erosion process of electrical switching contacts. Three-dimensional (3-D) surface measurement techniques provide an approach to investigate the geometric characteristics and volumetric erosion of electrical contacts. This paper presents a concrete data-processing procedure for evaluating volumetric erosion of spherical electrical contacts from 3-D surface measurement data using the defect removal method (DRM). The DRM outlined by McBride et al. [1] is an algorithm for evaluating the underlying form (prior to erosion) parameters of the surfaces with localized erosion and allowing the erosion characteristics themselves to be isolated. In this paper, a number of spherical electrical contacts that had undergone various electrical operations were measured using a 3-D surface profiler, the underlying form parameters of the eroded contacts were evaluated using the DRM, and then the volumetric erosions were isolated and calculated. The analysis of the correlations between the volumetric erosion and the number of switching cycles of electrical operation that the contacts had undergone showed a more accurate and reliable volumetric erosion evaluation using the DRM than that without using the DRM.
\end{abstract}

Index Terms-Contact erosion, electrical contact, form parameter, surface measurement.

\section{INTRODUCTION}

A $\mathrm{RC}$ erosion in electrical switching contacts fundamentally affects the reliability of the contact interface and, hence, the reliability of the switch itself. Mass change is generally used to measure the arc erosion; however this method does not describe the condition of the eroded contact surface profiles [2]. In recent years, three-dimensional (3-D) surface measurement techniques have been applied to investigate the arc erosion phenomenon and the condition of the eroded contact surface profiles. Additionally, using 3-D surface measurement techniques, the change in volume before and after electrical operation of the contacts, referred to as volumetric erosion, can be evaluated. This can lead to an improvement of understanding of the contact erosion process [3], [4].

Electrical contacts are produced with a variety of forms, including flat, spherical, and aspherical surfaces [3]. An important element within the study of the erosion characteristics is the evaluation and then removal of the underlying form (prior

Manuscript received January 27, 2004; revised July 21, 2005. This work was recommended for publication by Associate Editor M. Braunovic upon evaluation of the reviewers' comments.

D. Zhang is with the School of Engineering, University of Durham, Durham DH1 3LE, U.K. (e-mail: defen.zhang@hotmail.co.uk)

M. Hill and J. W. McBride are with the School of Engineering Science, University of Southampton, Southampton SO17 1BJ U.K.

Color versions of Figs. 2 and 4-9 are available online at http://ieeexplore. ieee.org.

Digital Object Identifier 10.1109/TCAPT.2006.880523 to erosion) of the eroded surface to allow the erosion characteristics themselves to be isolated. If the isolation method is inappropriate, it will cause incorrect analysis of the erosion characteristics and lead to an inaccurate evaluation of the volumetric erosion. Recently, an algorithm, referred to as the defect removal method (DRM), was outlined by McBride et al. [1]. This method enables the evaluation of the underlying form parameters of the surfaces with localized erosion from 3-D surface measurements and allows the erosion characteristics themselves to be isolated.

Electrical contacts having a nominally spherical surfaces, referred to as spherical electrical contacts, are commonly used in lower power switching devices [4]. Measurement of the volumetric erosion and surface characteristics of spherical electrical contacts using 3-D surface profilers has been addressed in previous research [2], [3], [5]. In this paper, the volumetric erosion of spherical eroded contacts from 3-D surface measurement data was evaluated using the DRM. First, the DRM was carried out to estimate the underlying form of the eroded contacts. After the correct underlying form has been estimated and removed from the measurement data, a flat datum surface remained and the erosion characteristics were isolated. The volumetric erosion was then calculated based on the isolated erosion data. Finally, the correlations between the volumetric erosion and the number of switching cycles of electrical operations that the contacts had undergone were compared and analyzed. This showed that the volumetric erosion could be evaluated more accurately using the DRM than without using this method.

\section{Defect Removal Method}

For any geometric form evaluation algorithm, it is assumed that measurement data are reasonably accurate and representative of the geometric elements concerned [6]. If sections of the data contain obvious outliers that are unrepresentative of the measured feature, the data should be preprocessed to remove these outliers [7]. The DRM is a data processing procedure for form fitting, which excludes data from heavily damaged areas of a surface prior to form fitting using a weighted least squares method.

In accordance with the applications within this paper, computer simulations were performed with spherical eroded contacts to demonstrate the operating procedure of the DRM. First, a pair of "idealized eroded contacts" were simulated, in which a part of an ideal sphere was used to simulate the body of the contacts and a paraboloid was superimposed around the crown of the ideal sphere to simulate the erosion, as shown in Fig. 1. It has been shown [1] that the anode of the contact generally gained mass while the cathode lost mass after electrical operations. The measurement results in this research clearly showed 

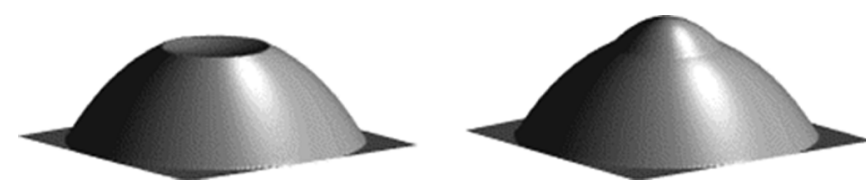

Fig. 1. Simulations of eroded electrical contacts.

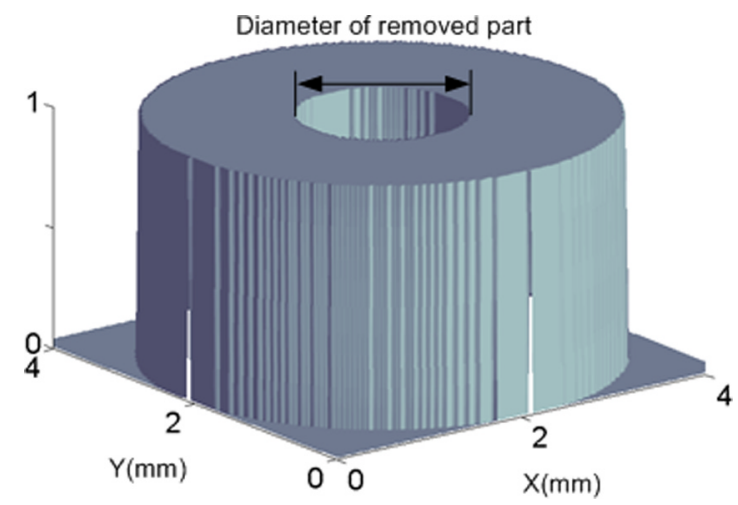

Fig. 2. Weighting function used for removing the eroded part.

that volumetric erosion was generally positive for the anode and negative for the cathode. Therefore, a convex paraboloid was applied to simulate an anode and a concave paraboloid for a cathode. To closely emulate the actual surfaces of the measured contacts, correlated Gaussian noise was superimposed on the "idealized eroded contact" surfaces to simulate the surface irregularity (form error and surface roughness).

To match the experimental data, the simulated measurement data for the electrical contacts covered a grid of $4.075 \mathrm{~mm} \times 4.075 \mathrm{~mm}$ in an array of $164 \times 164$ data points with a grid spacing of $\delta x=\delta y=0.025 \mathrm{~mm}$. The simulated spherical section had a radius of $2.375 \mathrm{~mm}$ and was centred at $(0,0,1.55)$. The eroded part covered a circular area with a radius of $0.6 \mathrm{~mm}$ in the $x y$-plane, which corresponded to most of the eroded contacts measured. The autocorrelated Gaussian irregularity superimposed on the "idealized eroded contact" surfaces had a correlation length of $0.2 \mathrm{~mm}$ with varied amplitudes (standard deviation $\sigma=5,10$, and $15 \mu \mathrm{m}$ ).

If the sphere was fitted with all the data, including the parabolic part as shown in Fig. 1, the fitting parameters would deviate from its nominal values of the contact underlying form due to the deformed data (simulated by the paraboloid surface). To remove the deformed data, a weighted least squares method was applied by designing a weighting function, in which the weight value of the paraboloid data was set to be " 0 " and the weight value for the other data was set to be "1," as shown in Fig. 2. In a real application, the bounds of the eroded region were not known a priori. Thus, the area with a weight value of " 0 " was increased gradually, i.e., the damaged data were removed incrementally. When all the eroded data were removed, the fitting radius and the central coordinate approached the real values. The procedure was realized with the simulated cathode eroded contact, as shown in Fig. 3.

The parameters that were evaluated in the test cases were the centre coordinate, the spherical radius and the residual root mean square (RMS). In the test cases, the standard deviation of the Gaussian irregularity $(\sigma)$ superimposed on the contact surfaces were, respectively 5, 10, and $15 \mu \mathrm{m}$. Because the defects occurred around the crown and were assumed to be symmetric about the $z$-axis, they affected the fitting radius and the $z$ coordinate of the sphere center, but did not affect the coordinates of the centre in the $x$ - and $y$-axes. For simplicity, only the fitting radius, the $z$ coordinate and the residual RMS were shown Fig. 3. In this figure, the radius of the removed part (in the horizontal axis) corresponded to the radius of the central "hole" shown in Fig. 2.

For the three test cases shown in Fig. 3, as the radius of the removed part increased, more and more deformed data were removed, and the fitting radius and the central coordinate approached the real values gradually. When the radius of the removed part reached a certain value $(0.6 \mathrm{~mm}$ in this case), all the defective data were eliminated. At this point, the clear plateaux were apparent in the graphs of all three tests. Therefore, it was clear that the defects were encompassed within that radius of $0.6 \mathrm{~mm}$ in these cases and this point was identified by the turning point on the graph of the residual RMS. Corresponding to this turning point, the parameters of the underlying form of the sphere were determined.

\section{EXPERIMENTAL SYSTEMS AND METHODS}

An experimental device has been developed in previous research [8] to simulate the switch operations for exploring erosion of electrical contacts after undergoing various operations. This device allows investigation of a large number of variables in a limited time period. The main parameters to be investigated include contact materials, number of test cycles, and current loading etc. Measurement of the volumetric erosion and surface characteristics of spherical electrical contacts has been addressed in previous research. In that research, stylus-based surface profilers have been found to be suitable for the 3-D measurement of small, partial spherical surfaces of electrical contacts [1], [2], [5]. In this study, Silver Cadmium Oxide (AgCdO) contacts with nominally spherical surfaces were subjected to various electrical operations using the switch operation experimental device. Their surface profiles were then measured using a surface profiler, The Form Talysurf, for volumetric erosion evaluation.

\section{A. Switch Operation Experiments}

A switch operation experimental apparatus described above is used to simulate the switch operations under alternating current (ac) conditions of $240 \mathrm{~V}$ and $50 \mathrm{~Hz}$. A computer controlled stepper motor is used to vary the opening velocity of the contacts between $0.01 \mathrm{~m} / \mathrm{s}$ and $1 \mathrm{~m} / \mathrm{s}$, as well as synchronizing the opening of the contacts with respect to the ac current cycles with an accuracy of $0.1 \mathrm{~ms}$; identified as the point-to-wave (POW). The POW is defined in terms of the point at which the contacts open relative to the current zero, as the current goes positive. A $1 \mathrm{~ms}$ POW is an operation where the contacts open $1 \mathrm{~ms}$ after current zero, $10 \mathrm{~ms}$ later, thus producing an arc of approximate 9 ms duration, since the arc is extinguished at the second current zero, $10 \mathrm{~ms}$ later. In the switch operation experiments, the tested contacts were used only to interrupt the circuit and the contacts 

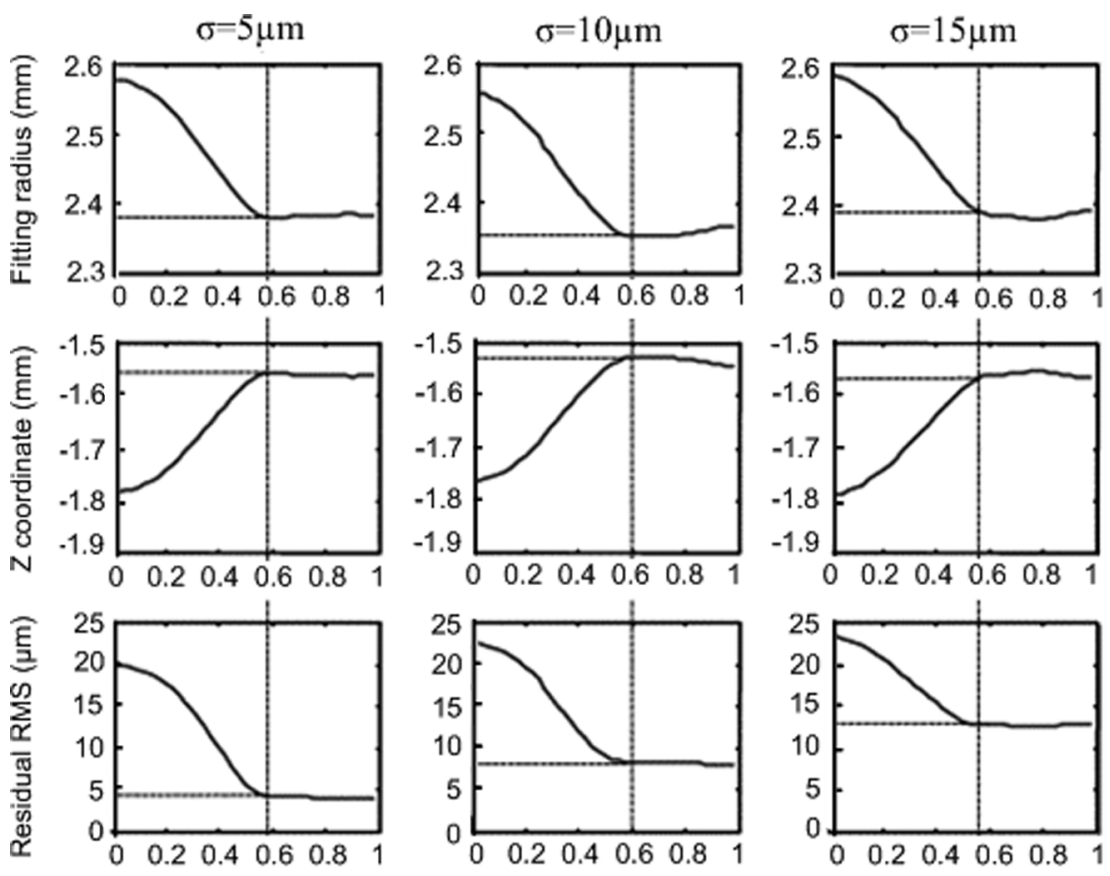

Radius of removed part $(\mathrm{mm})$

Radius of removed part $(\mathrm{mm})$
Radius of removed part $(\mathrm{mm})$

Fig. 3. Ppplication of the DRM on simulation data.

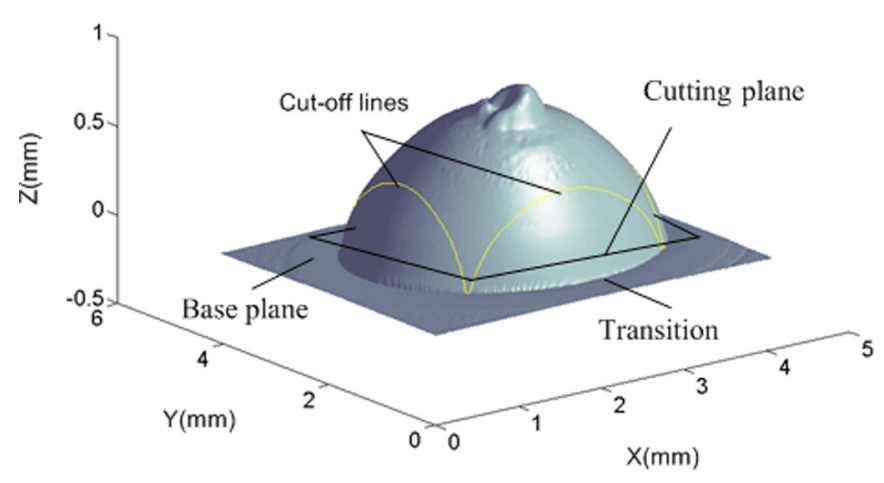

Fig. 4. Eroded contact with cutting-plane and cut-off lines.

were only operated on the positive half cycle, with the anode as the moving contact.

\section{B. Three-Dimensional Surface Measurements}

The Form Talysurf is a stylus based surface profiler for measurement of form and surface texture of components. This instrument operates by accurately monitoring the vertical displacement of the stylus ( $z$-axis), as it moves across a surface which is formed by two mutually perpendicular axes ( $x$-axis and $y$-axis). A Michelson interferometer is used to monitor the stylus movement in the $z$-axis. The measured results are reported in a Cartesian coordinate system. The resolution of the system in vertical axis is up to $10 \mathrm{~nm}$ and the measurement range is up to $6 \mathrm{~mm}$, making the system ideal for the precision measurement of curved surfaces. The measurements of the electrical contacts in this study were based on a grid with a spacing

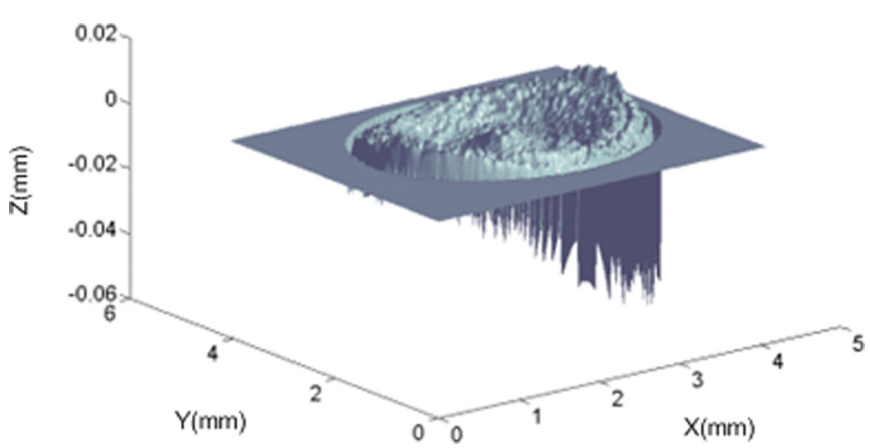

Fig. 5. Residual errors after fitting a new contact to a sphere when preconditioned with a $0.08-\mathrm{mm}$ high cutting-plane.

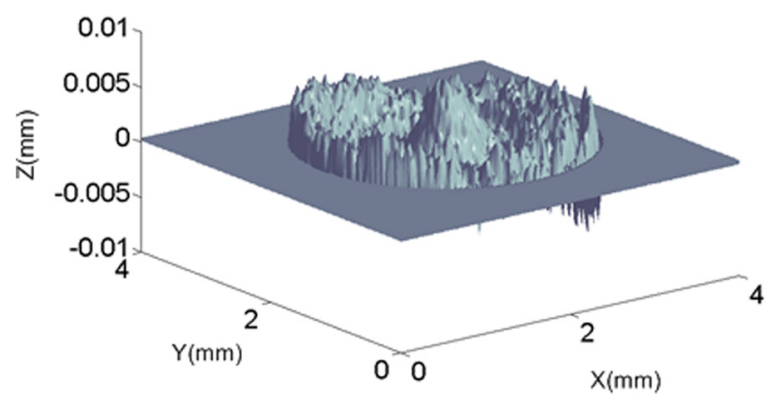

Fig. 6. Residual errors after fitting a new contact to a sphere when preconditioned with a $0.28-\mathrm{mm}$ high cutting-plane.

$(\delta x, \delta y)$ of $25 \mu \mathrm{m}$. The measured surface was described by up to 27000 discrete points, in an array of $164 \times 164$ data points. The scan area covered a square of $4.075 \mathrm{~mm} \times 4.075 \mathrm{~mm}$ in the $x y$-plane. 
TABLE I

Fitting Results After Data Preonditioning Using Cutting-Planes With Different Heights

\begin{tabular}{|c|c|c|c|c|}
\hline $\begin{array}{c}\text { Height of } \\
\text { Fitting results }\end{array}$ & $\begin{array}{c}\text { Mean of radius } \\
(\mathrm{mm})\end{array}$ & $\begin{array}{c}\text { STD of radius } \\
(\mu \mathrm{m})\end{array}$ & $\begin{array}{c}\text { Range of the } \\
\text { residual error } \\
(\mu \mathrm{m})\end{array}$ & $\begin{array}{c}\text { Residual RMS } \\
(\mu \mathrm{m})\end{array}$ \\
\hline $0.08 \mathrm{~mm}$ & 2.330 & 5.1 & 60 & 5.2 \\
\hline $0.28 \mathrm{~mm}$ & 2.375 & 3.0 & 15 & 2.3 \\
\hline
\end{tabular}

\section{ISOLATING EROSION OF ERODED CONTACTS USING THE DRM}

\section{A. Data Preconditioning for the DRM}

As described above, the measurement of the electrical contact covered an area of $4.075 \mathrm{~mm} \times 4.075 \mathrm{~mm}$ in the $x y$-plane. The measurement data included the whole contact surface and part of the base plane of the contacts, as shown in Fig. 4. Therefore, before fitting the surface measurement data of a contact onto a sphere, data preconditioning was needed to remove the measurement data representing the base plane.

In previous research [3], [5], cutoff lines shown in Fig. 4 were used for the data preconditioning to remove the measurement data representing the base plane. Although this method was visual and simple to realize in the computer program, a part of useful information representing the spherical surface was also lost. In this research, a method known as the "cutting-plane method" was applied to remove the data of the base plane and also the deformed data on the transition between the contact body and base plane.

1) Cutting-Plane Method: It was found from Fig. 4 that, if a plane which was parallel to and higher than the base plane was used to cut a measured contact and the data above the plane were kept for use with the DRM, almost all of the data on the spherical surface remained. Similar to the DRM, this was realized using a weighting function in conjunction with the least squares method. The weighting function was designed such that weight values of the data above the cutting-plane were " 1 " and the weight values of below this plane were " 0 ." It was also found in Fig. 4 that there was deformation in the transition between the contact body and the base plane, which might be caused by mechanical processes. A necessary step to remove all deformed data in the transition and keep more useful data for fitting the data to a sphere was to determine an appropriate high of the cutting-plane.

2) Determination of the Height of the Cutting-Plane: An appropriate height of the cutting-plane was determined, dependent on the extent of the deformation in the transition between the contact body and the base plane. It was obvious that electrical contacts produced by the same machining process had similar deformation. Thus, measurement data of a set of 17 new contacts were analyzed to determine the height of the cutting-plane that would be appropriate for all measured contacts, including the new and eroded ones.

For the 17 samples, cutting-planes with varied height (from 0.08 to $0.28 \mathrm{~mm}$ higher than the base plane) were used for producing the weighting function. To eliminate the influence of the surface irregularity of the base planes, the cutting-plane height was determined as the distance from the cutting-plane to the

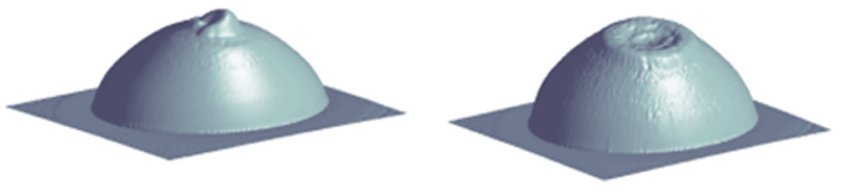

Fig. 7. Pair of anode and cathode contacts after 10000 switching cycles at 14.7-A, 1-ms POW, and opening velocity of $0.1 \mathrm{~m} / \mathrm{s}$.

upper-embraced plane fitted through the base plane data. It was found that, the fitting results from the same measurement data showed substantial differences using cutting-planes with different heights for data preconditioning. The mean and the standard deviation of the fitting radii of the 17 samples, the range of the residual error, and the residual RMS, were analyzed to determine the appropriate height of the cutting-planes.

Fig. 5 showed the residual errors after fitting the measurement data of a new electrical contact to a sphere, when preconditioned with a 0.8 -mm high cutting-plane. This figure suggested that the range of the residual errors was larger than 0.06- $\mathrm{mm}$ and a large number of the deformed data in the transition were included for sphere fitting. Thus, the cutting-plane was raised gradually up to $0.28-\mathrm{mm}$ higher than the base plane. It was shown in Fig. 6 that the range of the residual errors was less than $0.015 \mathrm{~mm}$, when preconditioning with a $0.28 \mathrm{~mm}$ high cutting-plane. The residual errors in this figure were considered to be caused by the surface irregularity rather than the deformation data; therefore, it was deemed that all the deformed data in the transition were excluded. The fitting results for these two cases are shown in Table I. It was established that the measurement uncertainty of the radii of the 17 samples was better for the data that has been preconditioned with a 0.28 - $\mathrm{mm}$ high cutting-plane than that of the data preconditioned with a $0.8-\mathrm{mm}$ high cutting-plane. In following sections, all measurement data of the eroded contacts were preconditioned by using the 0.28 - $\mathrm{mm}$ high cutting-plane before undergoing further data processing.

\section{B. Isolation of the Volumetric Erosion}

A number of spherical electrical contacts, which has undergone various operations using the switch operation experimental device described above, have been measured with The Form Talysurf surface profiler. After the data preconditioning described above, the measurement data were processed using the DRM. The data processing results of a typical pair of eroded anode and cathode, as shown in Fig. 7, are presented in Fig. 8. It can be seen that the trends on the graphs in the real measurement data are less regular than was the case with the simulated data. It is likely that this is due, in part, to the surface irregularity of the measured contacts. Zhang et al. [9] have 

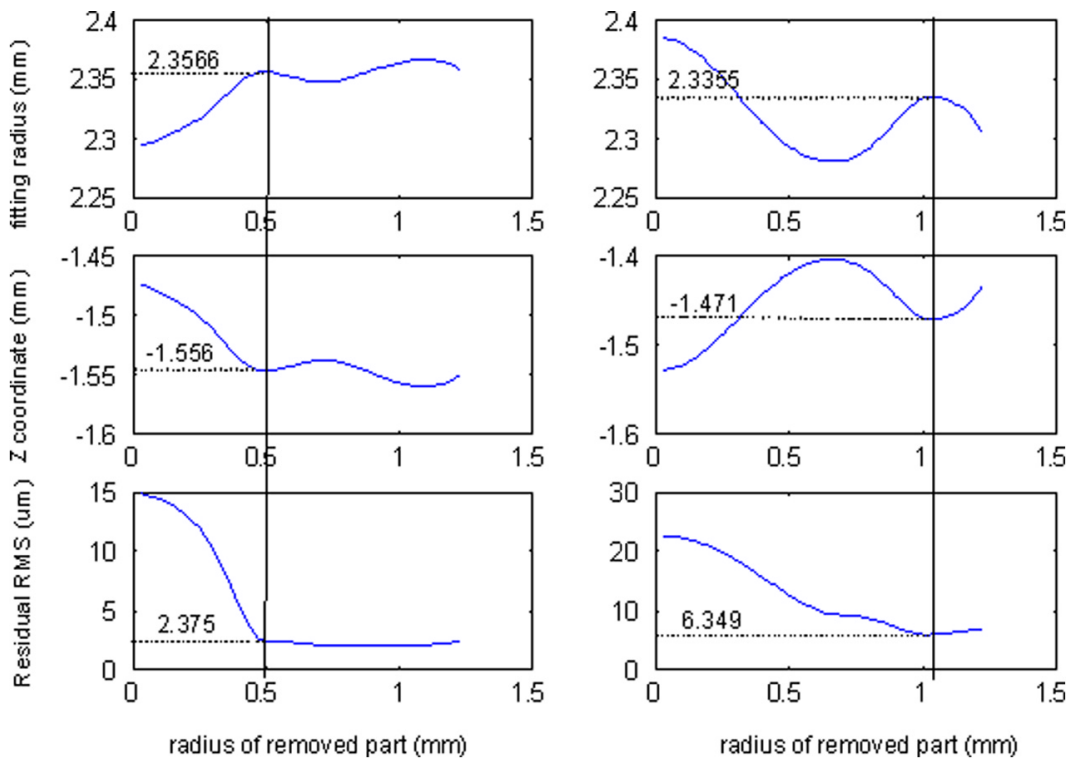

Fig. 8. Application of the DRM on the measurement data of a pair of eroded electrical contacts.

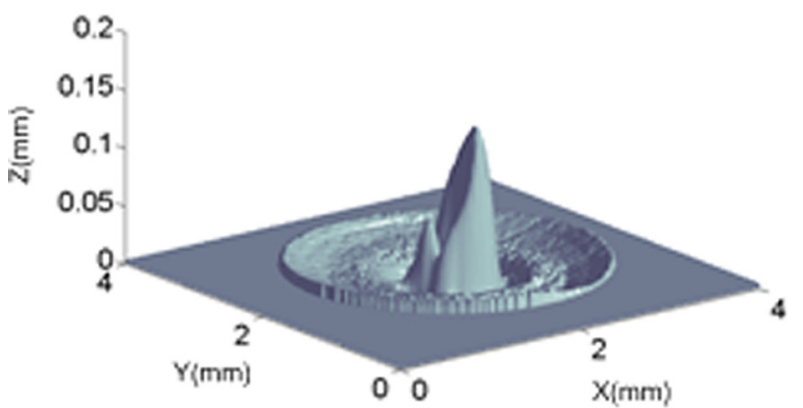

Fig. 9. Isolated erosion of the eroded contacts shown in Fig. 8.

found that surface roughness and form error produced a deviation on the evaluation of the form parameters. Additionally, arc erosion generally occurs in the central parts of contact surfaces, but there are light defects on the other parts of the contact surfaces, which may have an effect on the algorithm. However, once again the turning points occurred close to the observed plateaux in the residual RMS. Corresponding to these turning points, the parameters of the underlying form of the eroded contacts were estimated. After removal of the underlying form from the measurement data, a flat datum surface remained and the erosion was isolated as shown in Fig. 9. Based on the data of the isolated erosion, the volumetric erosion can be evaluated.

\section{VOLUMETRIC EROSION EVALUATION}

\section{A. Numerical Algorithms for Volume Evaluation}

Algorithms for estimating volumetric erosion have been detailed in [3], including the average of points technique, two-dimensional (2-D) linear approximation, the 3-D trapezium technique, simplified 3-D linear approximation, and the 3-D Simpson's rule. Among these methods examined, the averaging techniques were found to underestimate the volume. Studies

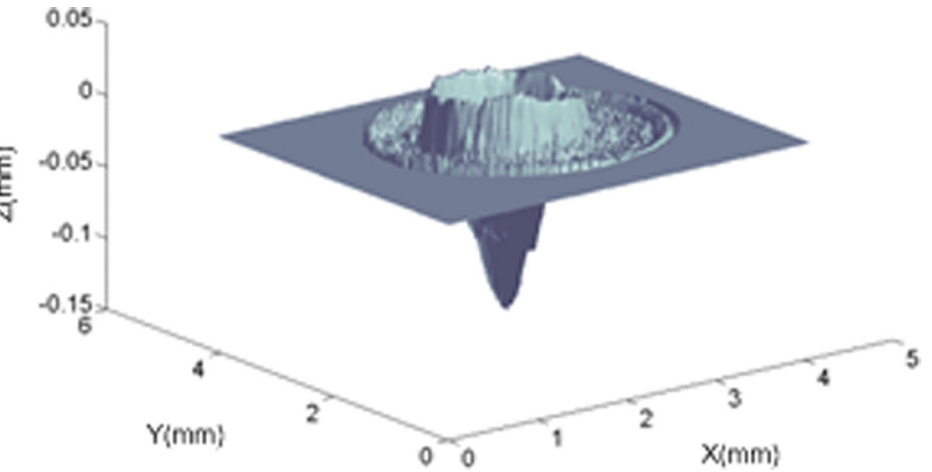

showed that the 3-D Trapezium technique was accurate but computational inefficient, whereas the 3-D Simpson's rule was proved to be the most accurate in determining volumetric erosion of the electrical contacts, and computationally efficient.

The 3-D Simpson's Rule splits the data into groups of three, evaluates the area under each group and then sums these areas. At the end of each trace of data, there may be one extra point, which cannot be used for a Simpson approximation, for example, point 164 since the groups are points 1 to 3,3 to $5, \ldots$, 161 to 163 . In this case, the area is calculated using points 163 and 164 as a trapezium and adding this to the area calculation. The formula is shown in

$$
V=\sum_{y=1}^{T / 2}\left[\frac{A_{2 y-1}+4 A_{2 y}+A_{2 y+1}}{2}\right] \cdot \delta_{y}
$$

where $V$ is the volumetric erosion; $A_{y}=\left[\left(\left(Z_{1, y}+Z_{P, y}\right) / 2\right)+\right.$ $\left.\sum_{x=2}^{P-1} Z_{x, y}\right] \cdot \delta_{x} ; T$ is the number of traces in a sample; $P$ is the number of points per trace; $\delta_{\mathrm{x}}$ and $\delta_{\mathrm{y}}$ are the data point spacings in the $x$ - and $y$-directions, respectively. 


\section{B. Volumetric Erosion Evaluation Using the DRM}

Volumetric erosion of a number of pairs of eroded anodes and cathodes have been processed based on the isolated erosion data using the DRM. For comparison, the volumetric erosion evaluation results from the method that involved direct estimation of the form parameters of the eroded contact, without using the DRM, were processed as well.

Volumetric erosion derived from the same measurement data sets with and without use of the DRM was significantly different. For the sample of the anode shown in Fig. 7, the volumetric erosion evaluated after using the DRM was positive $\left(18.62 \times 10^{-3} \mathrm{~mm}^{3}\right)$, whereas the erosion evaluation without using the DRM was negative $\left(-3.260 \times 10^{-3} \mathrm{~mm}^{3}\right)$. For the cathode sample, the volumetric erosion obtained using the DRM was negative $\left(-61.9 \times 10^{-3} \mathrm{~mm}^{3}\right)$, but the erosion derived prior to defect removal was positive $\left(8.32 \times 10^{-3} \mathrm{~mm}^{3}\right)$. Observing the geometric shapes of these two eroded contacts, as shown in Fig. 9, it was obvious that the volume of the anode increased and the volume of the cathode decreased after the electrical operations. The volumetric erosion derived using the DRM were more in agreement with the visual estimates.

\section{Volumetric Erosion and Switching Cycles}

To explore the relationship between the volumetric erosion and the switching operation, the volumetric erosion of the cathode and anode as a function the number of switching cycles of the electrical operation are shown in Figs. 10 and 11. The experimental condition was under a circuit current of 14.7-A, 1-ms POW. The opening velocity was 0.1 and $0.8 \mathrm{~m} / \mathrm{s}$, respectively, for these two cases. It was clearly shown in Figs. 10 and 11 that the anodes always gained and the cathodes always lost volume after using the DRM. It was also shown that the volumetric erosion evaluated using the DRM had a good correlation with the number of switching cycles of electrical operations, which matched the previous research results [3], [4]. However, the volumetric erosion evaluated without using the DRM did not show obvious trends along the number of switching cycles of the electrical operations. In some cases, it even showed that the cathode gained and the anode lost volume.

Comparing the graphs in Figs. 10 and 11, it was found that the volumetric erosion evaluated using the DRM showed a similar relationship with the number of operations. However, in the case of the higher velocity $(0.8 \mathrm{~m} / \mathrm{s})$, the magnitudes of the volumetric erosion were reduced when compared with the case of the lower velocity $(0.1 \mathrm{~m} / \mathrm{s})$. This meant that the higher opening velocity reduced the effects of the arc material deposited on to the contact support, which corresponded to the previous research results [3]. These comparison and analyses proved that the DRM produced an accurate underlying form evaluation and further, resulted in an accurate and reliable evaluation of the volumetric erosion of the eroded contacts.

\section{CONCLUSION}

Volumetric erosion has been regarded as a significant index for studying the erosion process of electrical contacts. Three-dimensional surface measurement techniques provide an approach to investigate the geometric characteristics and

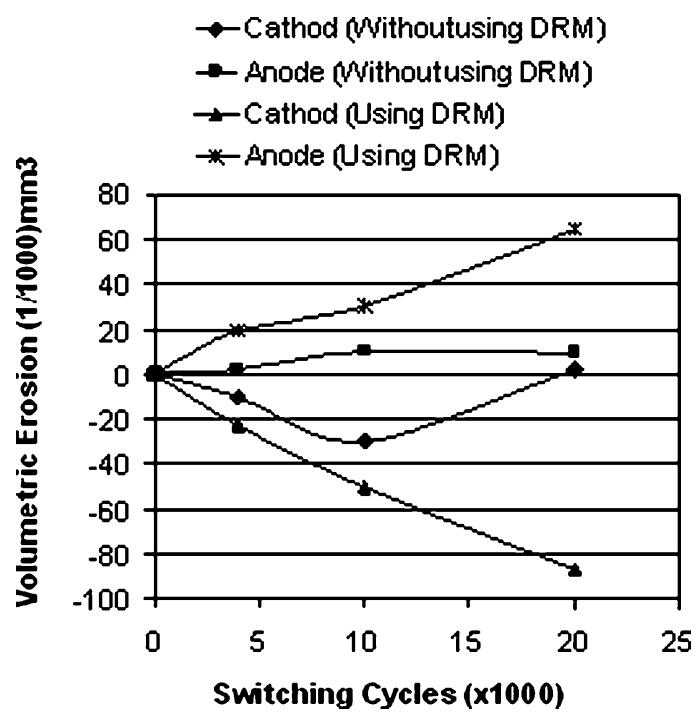

Fig. 10. Cathode and anode net volumetric erosion compared with the number of switching cycles at 14.7-A, 1-ms POW and opening velocity of $0.1 \mathrm{~m} / \mathrm{s}$.

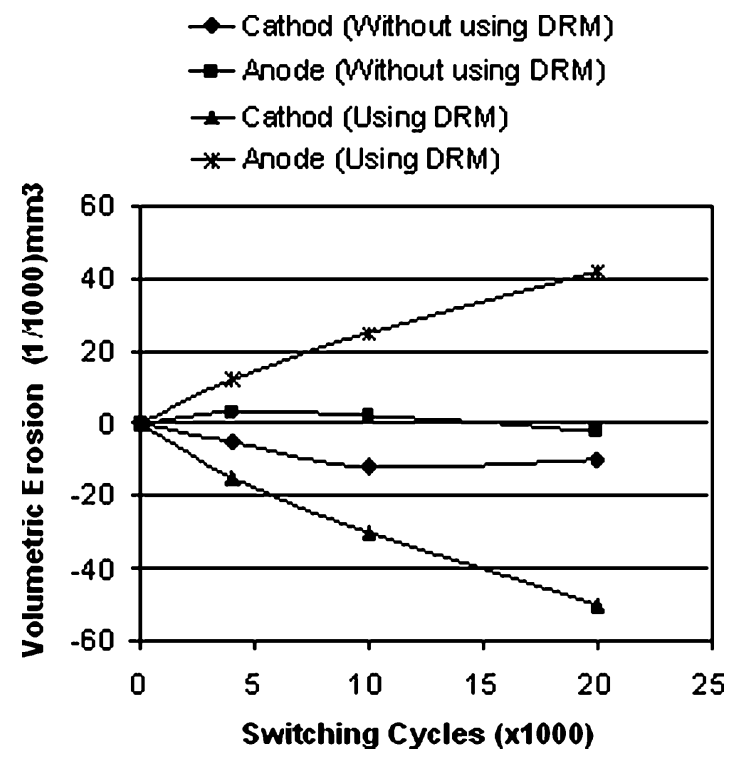

Fig. 11. Cathode and anode net volumetric erosion compared with the number of switching cycles at 14.7-A, 1-ms POW and opening velocity of $0.8 \mathrm{~m} / \mathrm{s}$.

volumetric erosion of electrical contacts. An important element within the evaluation of volumetric erosion from 3-D surface measurements is the fitting and removal of the underlying form of the eroded surface to allow the erosion characteristics themselves to be isolated. The DRM provides this capability.

In this paper, the DRM was applied to measurement data of eroded spherical electrical contacts. The practical applications showed that the DRM was able to identify the damaged parts and find the underlying form parameters of the damaged surfaces, although surface error produced some disturbances. After the correct underlying form was removed, the volumetric erosion was isolated, and consequently, the accurate and reliable volumetric erosion was obtained. Based on the comparison and analysis of the volumetric erosion of the eroded contacts that had undergone various electrical operations, it has been found that the evaluation of the volumetric erosion using the DRM showed 
more clear trends, in terms of the number of electrical operations carried out, than that obtained without using the DRM. This analysis has proven that the DRM is an essential process for the volumetric erosion evaluation of eroded contacts.

Although the emphasis of this paper is on evaluation of the volumetric erosion of spherical electrical contacts applying the DRM, the extended purpose is to present a concrete dataprocessing procedure for the evaluation of volumetric erosion of other types of electrical contact using 3-D surface measurement data.

\section{REFERENCES}

[1] J. W. McBride, M. Hill, and D. Zhang, "A feature extraction method for the assessment of form parameters of surfaces with defects," Wear, vol. 256, pp. 243-251, Feb. 2004.

[2] J. W. McBride, A Review of Volumetric Erosion Studies in Low Voltage Electrical Contacts The Institute of Electronics, Information and Communication Engineers, Tech. Rep. IEICE EM2001-45, Sept. 2001.

[3] J. W. McBride, K. J. Cross, and S. M. Sharkh, "The evaluation of arc erosion on electrical contacts using three dimensional surface profiles," IEEE Trans. Compon., Packag. Manufact. Technol., vol. 19, no. 4, pp. 87-97, Dec. 1995.

[4] J. W. McBride, "The volumetric erosion of electrical contacts," IEEE Trans. Compon., Packag. Manufact. Technol., vol. 23, no. 2, pp. 211-221, June 2000.

[5] M. Jung, K. J. Cross, J. W. McBride, and M. Hill, "A method for the selection of algorithms for form characterization of nominally spherical surface," Precision Eng., vol. 24, pp. 127-138, Oct. 2000.

[6] A. B. Forbes, "Validation of assessment software in dimensional metrology," in Proc. SPIE Int. Soc. Opt. Eng., 1993, pp. 28-36.

[7] Assessment of Position, Size and Departure from Nominal Form of Geometric Features, British Std. BS7172, 1989.

[8] J. W. McBride and S. M. Sharkh, "The effect of contact opening velocity and the moment of contact opening on the arc erosion of $\mathrm{AgCdO}$ contacts," IEEE Trans. Compon., Packag. Manufact. Technol., vol. 17, no. 1, pp. 2-7, Mar. 1994

[9] D. Zhang, M. Hill, J. W. McBride, and J. H. Loh, "The form evaluation algorithms," in Proc. 6th Int. Conf. Laser Metrol. Mach. Perf., Huddersfield, U.K., 2003, pp. 273-283.

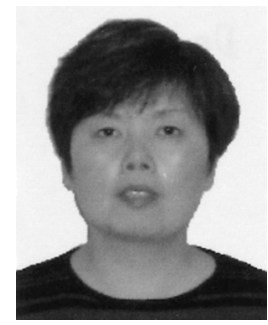

Defen Zhang received the Ph.D. degree in surface metrology in mechanical engineering from the University of Southampton, Southampton, U.K., in 2003.

She is currently a Research Fellow in the School of Engineering, University of Durham, Durham, U.K. Her research experiences and interests are mainly in coordinate metrology, concerning algorithm study for 3-D tolerance analysis, measurement uncertainty analysis, large-scale metrology techniques, and sensor techniques.

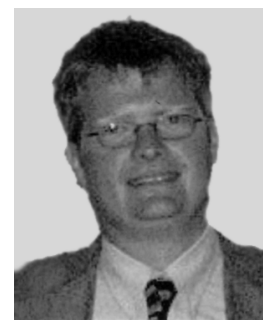

Martyn Hill received the Ph.D. degree from the Institute of Sound and Vibration Research, University of Southampton, Southampton, U.K., in 2004.

He then moved to the Mechanical Engineering Department where he carried out research into parameter estimation techniques for noninvasive biomedical measurement. He was appointed Lecturer in 1990 and is now a Reader in the Electromechanical Research Group, University of Southampton. His current research interests are in the areas of acoustics and transducers and metrology.

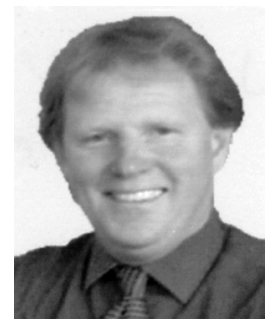

John W. McBride received the M.S. degree in aeronautical engineering from the University of Southampton, Southampton, U.K., in 1978 and the $\mathrm{Ph} . \mathrm{D}$. degree in electrical contact phenomena from Plymouth University, Plymouth, UK, in 1986.

From 1985 to 1987 , he lectured in the Mechanical Engineering Department, Plymouth University. Since 1987, he has been a Lecturer, Senior Lecturer, Reader, and since 2002, Professor in instrumentation and measurement in the School of Engineering Science, University of Southampton. His main research interests include electrical contacts, metrology, and instrumentation.

Dr. McBride is a Chartered Engineer and Chair of the Electro-Mechanical Research Group. 